\title{
Editorial
}

\section{Der Nationalstaat zwischen globaler Ökonomie, regionaler Blockbildung und regionalistischem Separatismus}

Als während und nach dem Zweiten Weltkrieg die neue Welt(wirtschafts)ordnung (als "grand design «) konzipiert wurde, war Freihandel das deklarierte Ziel. Das 1947 zustandegekommene Allgemeine Zoll- und Handelsabkommen (GATT) verpflichtete die Mitgliedsländer auf den Abbau von Zöllen und auf die Einhaltung der Meistbegünstigung. Auch das wenige Jahre zuvor 1944 gegründete Internationale Währungssystem mit dem Regelwerk fixer Wechselkurse sollte den Handel erleichtern und so ein Positivsummenspiel eröffnen, an dessen Ende Wachstum und Wohlstand aller Welthandelspartner zugenommen haben. Die Gefahr eines desaströsen Abwertungswettlaufs wie noch zu Beginn der 30er Jahre sollte ein für alle Mal gebannt sein.

Die Begründung der Freihandelsordnung lieferten nicht nur die schockierenden Erfahrungen nach der Krise von 1929, als der Weltmarkt in kurzer Zeit zusammenbrach, sondern auch das ehrwürdige Theorem der komparativen Kostenvorteile von David Ricardo. Die Besten der Ökonomenzunft haben es differenziert, relativiert und modernisiert und die Fahne des Frei- handels geschwungen. Trotzdem blieb das Theorem von heftiger Kritik nicht verschont. Wenn man von den Sarkasmen absicht, mit denen beispielsweise Marx sich über die britischen Freihändler mokierte, markiert insbesondere Friedrich List die Gegenposition: Die Freihandelsdoktrin sei die Lehre der industriell fortgeschrittensten, überlegensten Nation und für all jene unbrauchbar, die gezwungen sind, im Medium des Weltmarkts industrielle Entwicklung nachzuholen und konkurrenzfähig $z u$ werden. Nachholende Industrialisierung sei nur hinter den Mauern von Schutz- und Erziehungszöllen möglich, also indem bewußt und selbstbewußt die Regeln des Freihandels außer Kraft gesetzt werden.

Nach dem Zweiten Weltkrieg wird die Kontroverse fortgesctzt. In den diversen Zollrunden des GATT wird der Versuch gemacht, tarifäre und nichttarifäre Handelshemmnisse abzubauen, also dem Prizip des Freihandels auf Erden näherzukommen, auch wenn die größten Ideologen des Freihandels in der Praxis heftige Protektionisten sind (in der EG, aber auch in den USA). Wenn es hingegen um entwicklungspolitische Stra- 
tegien in den Entwicklungsländern geht, wird weniger auf Ricardo denn auf Friedrich List und seine nationalökonomische Lehre von den »produktiven Kräften« rekurriert, bis hin zu den Konzepten einer partiellen und temporären Dissoziation nationaler Wirtschaftsräume vom Weltmarkt der 70er Jahre. Wenn Ricardo und List gegeneinander ausgespielt werden, geht es daher auch um das Verhältnis von Ökonomie und Politik. Die Freihandelsdoktrin ist die Theorie der globalen Ökonomie, während die Listsche Lehre von der autozentrierten Entwicklung das Konzept einer nationalstaatlichen Politik zur Förderung der produktiven Kräfte darstellt. List wendet sich daher gegen den »Kosmopolitismus « der Freihändler und plädiert für eine »Nationalökonomie« in der impliziten Annahme von der an den Nationalstaat gekoppelten Souveränität. Daß dies aus der Sicht der letzten Jahrzehnte des 20. Jahrhunderts fragwürdig ist, wird von Rolf Knieper (vgl, seinen Aufsatz in diesem PROKLA-Heft) zum Thema gemacht.

Die Kontroverse zwischen Freihändlem und Schutzzöllnem ist sehal geworden. Denn in den $80 \mathrm{er}$ Jahren sind auf dem Weltmarkt neben der globalen, kosmopolitischen Ökonomie und der nationaleu Politik intermediäre Einheiten entstanden: regionale Handels- und Wirt schaftsblöcke. Nationale Ökonomien haben sich zu Freihandelszonen oder Zollunionen zusammen- geschlossen, also den Versuch unternommen, wenn schon nicht im globalen Raum unter der Ägide des GATT insgesamt, so doch in einem regional überschaubaren Block den Prinzipien des Freihandels näherzukommen und dessen wohlfahrtssteigernde Effekte einzuheimsen. Unter den Freihandelszonen im westlichen Afrika, in Zentralamerika, im andinen Raum, im Pazifik und Südostasien ist die Europäische Gemeinschaft sozusagen der Doyen nicht nur wegen des inzwischen hohen Alters - die EG ist immerhin im Jahre 1957 entstanden - sondern auch wegen der politischen und ökonomischen Bedeutung, die sie inzwischen in die Waagschale bringen kann. Die Wirtschaftsblöcke Asiens, Afrikas und Lateinamerikas bringen es gerade auf knapp 11\% des Welthandels, während allein die EG im Jahre $199041,4 \%$ des Welthandels auf sich vereinigt. Die Blockbildung der Kleinen ist nicht besonders überzeugend. Der schon geringe Anteil am Welthandel ist im vergangenen Jahrzehnt noch geringer geworden. Dieses Resultat läßt darauf schließen, daß die Bildung von Handelsblöcken für sich genommen ebenso wenig ein Schlüssel zum ökonomischen Erfolg ist wie ein globaler Freihandel oder nationalstaatlich forcierte, autozentrierte Entwicklungsstrategien. Erst wenn Synergieeffekte ausgelöst werden, lohnt sich offenbar die Blockbildung. Nicht gerade in Übereinstimmung mit dem Theorem von 
den komparativen Kostenvorteilen ergeben sich offensichtlich Synergieeffekte am ehesten, wenn die beteiligten Ökonomien ähnlich hohe Entwicklungsniveaus und ähnliche Produkipaletten aufweisen. Der intraindustrielle Handel innerhalb der EG oder auch innerhalb der OECDWelt ist offensichtlich dynamischer als der Austausch zwischen komplementären Produktgruppen, beispielsweise zwischen Industriegütern und Rohstoffen innerhalb eines Hanclelsraums.

Die kleinen Handelsblöcke könnte man, von Europa aus betrachtet, durchaus vernachlässigen. Lateinamerika ist am Ende der 80er bzw. an Anfang der 90er Jahre ohnehin nur mit $4,2 \%$ an den Importen der OECD beteiligt, Afrika nur mit $1,9 \%$, Osteuropa lediglich mit $2,1 \%$ und selbst Asien/Ozenanien bringt es nur auf $5,4 \%$. Infolgedessen richtet sich das Augenmerk, wenn von Regionalisierung der Weltwirtschaft oder Blockbildung die Rede ist, ausschließlich auf die Blöcke, die um die höchstentwickelten Indlustrieländer herum existieren oder im Entstehen sind: Die EG hat mit dem Maastrichter Vertrag einen mächtigen Schritt zur Vertiefung der Integration projektiert, die USA versuchen ihre mit Kanada 1989 geschaffene Freihandelszone auf Mexiko auszudehnen; der Vertag ist Ende 1992 beschlossen, das Ratifizierungsverfahren ist eingeleitet worden (zur NAFTA vgl. den Aufsatz von Enrique Dussel). Und Japan reiht die asiatischen Staaten wie einen eher informellen Kranz um sich, da es eine formalisierte Freihandelszone oder Zollunion über bilaterale Beziehungen hinaus höchstens in Ansätzen gibt. Am Ende der Blockkonfrontation zwischen dem »freien Westen« insgesant und dem »sozialistischen Lager« löst sich die westliche Einheit, sofern sie je existiente, in eine »Triade« auf. Nicht globaler Freihandel ist deren regulierendes Prinzip sondem eine staatlich und suprastaatlich regulierte Konkurrenz in geopolitisch definierten Regionen, in denen es um industrielles Überleben geht.

Die Blockbildung in der OECDWelt ist eine wirksame Tendenz, die freilich durchaus Handelskonflikte ausiösen kann. Die jüngste Kontroverse zwischen $\mathrm{EG}$ und USA über Ölsaaten, Weizen und Weißweine oder zwischen Japan und den USA um Reisexporte hat gezeigt, daß regionale Handelshlöcke nicht geschlossen sind sondern interferierende Außenbeziehungen aufweisen, die schwierig zu handhaben sind. Das globale Prinzip des Freihandels oder die von Marx so bezeichnete »propagandistische Tendenz« des Weltmarkts, wirken weiter. Also gibt es jenseits der Blockbildung gemeinsame Interessen an der Existenz eines intemationalen und interregionalen Rahmens. Handels- und Wirtschaftsblöcke ersetzen das globale System und dessen Prinzipien der politischen Re- 
gulation nicht. Alle Blöcke, so konkurrierend oder »selbstgenügsam《 (Keynes) sic sich auch gebärden mögen, benötigen gemeinsame »öffentliche Güter«: ohne Versorgung mit Sicherheit, mit Rohstoffen und Energie auf hohem Niveau zu stabilen Konditionen und ohne wertbeständiges Geld auf dem Weltmarkt sind Marktprozesse nicht effizient. Sie sind also auf das angewiesen, was im »alten « US-amerikanischen Hegemonialsystem der Nachkriegszeit die hegemoniale Macht bereitzustellen vermochte (natürlich nicht onne ihren "Seignoragevorteil" geltend zu machen). Nach der sich schon Ende der 60er Jahre ankündigenden Erosion der hegemonialen Vormachtstellung der USA hat das »burden sharing des Trilateralismus der $70 \mathrm{er}$ Jahre ebensowenig funktionieren können wie die letztlich gefährlich naive Idee, daß mit der Freisetzung der Marktkräfte die "offentlichen Güter" quasi nebenbei produziert würden. Aber auch die nach dem Ende der bipolaren Ordnung siegestrunkene Annahme, daß die öffentlichen Güter »unipolar « von den USA bereitgestellt werden könnten, hat sich schon kurz nach dem während des Golfkonflikts angekündigten »unipolar moment « als eine trügerische Illusion herausgestellt.

Das »burden sharing « muß also auf den neuesten Stand gebracht werden. Bei der Herstellung von Sicherheit geht es nicht mehr um Sicherheit vor einer »kommunisti- schen Aggression « oder vor dem gefürchteten »Dominoeffekt « des Kollapses von Partnerländern im Bündnissystem, sondern um die Eindämmung der Negativfolgen der innerhalb der Triade so beeindruckenden ökonomischen und sozialen Entwicklung. Waffen zu produzieren und zu exportieren, ist ein gutes Geschäft, das den Wohlstand hebt. Wenn freilich die Käufer der Waffen zu mächtig werden und eine Bedrohung darstellen, gebietet es das gemeinsame Sicherheitsinteresse, die Waffenstaaten »zwangsabzurüsten«. Die Externalisierung ökonomischer Folgekosten industrielles Produktion ist eine ebenso naheliegende wie verbreitete Methode, un das Wohlstandsniveau zu halten. Wenn aber die externalisierten Unweltlasten in der einen oder anderen Form wieder zurückkehren, dann gebietet es das Sicherheitsinteresse, gemeinsame Vorkehrungen zu treffen. Wenn die Attraktivität der reichen Industriezonen wächst, weil sie reicher werden, während die nichtindustrialisierte Welt verarmt und daher Migrationsströme auslöst, dann gebietet es das Sicherheitsinteresse, Mauern und Zäune zu errichten - mit entsprechenden Rückwirkungen auf die Legitimation der »freiheitlichen Rechtsstaaten « nach innen (vgl. hierzu den Beitrag von Uli Jähner). Auch die gesicherte Rohstoff- und Energieversorgung liegt im gemeinsamen Interesse, da ja alle Industrieländer wegen ihrer »fossilistischen« Grundlage von 
strategischen Rohstoff- und Energieimporten abhängig sind oder in absehbarer Frist abhängig werden. Entscheidend ist dabei, daß die Versorgung zu akzeptablen Preisen sichergestellt wird, hängen davon doch der »Wohlstand der Nationen « und die Konkurrenzfähigkeit der Unternehmen $\mathrm{ab}$.

Schließlich ist die Regulation des Geldes als Weltgeld von gemeinsamem Interesse, jedenfalls solange trotz Regionalisierung das Welthandels- und Finanzsystem globalisiert bleiben. Hier liegt iibrigens der neuralgische Punkt der Regionalisierung der Weltwirtschaft. Denn spätestens seit den 60 er Jahren hat sich ein internationales Bankensy. stem mit globaler Reichweite herausgebildet, das, solange die Konvertibilität der Währungen Prinzip bleibt, nicht in Grenzen regionaler Wirtschaftsblöcke eingehegt werden kann. Dafür ist die Mobilität des zinstragenden Kapitals viel zu hoch. Es kennt kein Vaterland und keine Muttersprache und ist in New York ebenso zuhaus wie in Buenos Aires, Singapur oder Frankfurt, sofern die Rendite stimmt. Ein Weltgeld ist also ein funktionales Erfordernis der Weltwirtschaft. Doch gleichzeitig sind weder ein Weltstaat noch eine Weltzentralbank vorhanden, die es emittieren und regulieren könnten. Also werden nationale Währungen zum Weltgeld. Im Bretton WoodsSystem hatte der US-Dollar unangefochten die Funktion der Handels-, Reserve- und Leitwährung, war also das (einzige) Weltgeld. Seit dem Ende des Bretton WoodsSystems jedoch konkurrieren nationale Wahrungen mil anderen nationalen Währungen um die $»$ Vorliebe« der Geldvermögensbesitzer, ihre Vermögen in der jeweiligen Währung zu halten. Die Konkurrenz. nationaler Währungen leistet daher auch der Regionalisierung und Blockbildung in der Weltwirtschaft Vorschub, insofern nämlich keine prominente, nationale Währung mehr unangefochten die Funktionen des Weltgeldes ausïben kann.

Solange der Dollar der Fixstern war, um den die anderen Wäbrungen wie Planeten in starrer Umlaufbahn kreisten, konnten sich Währungsblöcke überhaupt nicht herausbilden; sie machten schlicht keinen Sinn. Als der Dollar freilich zu Begim der $70 \mathrm{er}$ Jahre seine hegemoniale Stellung verlor und gegenüber konkurrierenden Währungen wie der DM und dem Yen abgewertet werden mußte, nachdem das Vertraten in die Stabilität des Dollar enttäuscht worden war, wandten sich die privaten Geldvermögensbesitzer weltweit altemativen Währungen zu, um darin ihre Geldvermögen »sicher" zul parken. Neben dem Dollarraum entstanden so ein DMRaum und ein Yen-Raum. Die Währungskonkurrenz ist monetärer Schrittmacher der Regionalisierung der Weltwirtschaft. Die monetäre Triade von Dollar, Yen und DM (bzw. ECU, wenn man das europäische Währungssystem als ein weit 
fortgeschrittenes monetäres Einigungswerk in die Diskussion einbezicht) reflektiert also die Triade zwischen Westeuropa, Nordamerika und dem pazifischen Raum.

Da die großen Währungen aber wechselseitig frei konvertibel sind, hat die Blockbildung (bislang jedenfalls) keineswegs zur wechselseitigen Abschottung geführt, sondern mit der Währungskonkurrenz eher die realökonomische Konkurrenz belebt. Dieser Sachverhalt ist denn auch zum Gegenstand der unzähligen Untersuchungen über die »japanisch-amerikanische Herausforderung« (Seitz), die »lean-production« in Japan, den »Toyotismus usw. promoviert. Nur weil die Währungen frei konvertibel sind, und Handelsschranken zwar nicht belanglos, aber auch nicht prohibitiv sind, spielt der Vergleich zwischen der Population von Industrierobotern (in Japan Ende 1990 274.210; in den USA 41.304 und in der BRD 28.240), den Forschungs- und Entwicklungsausgaben am Sozialprodukt (in Japan 3,51\%; in den USA $3,26 \%$, in der BRD 3,70\%), den externen Forderungen und Verbindlichkeiten (die USA haben Ende 1990397 Mrd US\$ Schulden, Japan hat Auslandsaktiva in Höhe von 327 Mrd US\$, die BRD 355 Mrd US\$), dem Bestand von Direktinvestitionen (Ende 1990 USA: 421,5 Mrd US\$, Japan: 201,4 Mrd US\$, BRD: 114,5 Mrd US\$) etc. eine wesentiiche Rolle. Japan ist unter den OECD Ländern das einzige Land, das seinen Anteil von High-TechProdukten am Export zwischen 1980 und 1990 hat steigem können, und zwar von $15,3 \%$ auf $19,1 \%$. Die Bundesrepublik, aber auch die USA, sind auf diesem Sektor zurückgefallen (von $15,9 \%$ auf $14,6 \%$, bzw. von $25,1 \%$ auf $23,7 \%$ ). Japan ist Japan, die Staaten des pazifischen Beckens nehmen im Wettrennen mit den anderen Wirtschaftsblöcken nicht in der Staffel gemeinsam und gleichberechtigt mit Japan teil, sondern in der Etappe, wo sie für Japan komplementäre Funktionen übernehmen (vgl. daza den Artikel von Heribert Dieter in diesem Heft).

Ganz anders ist dies im Falle Westeuropas. Hier existiert ein vergleichsweise homogener Wirtschaftsraum, der über cine hochentwickelte Infrastruktur, exzellente Qualifikationen der Arbeitskraft und kompetente Institutionen verfügt, die für eine europaweite »diversified quality production « höchst funktional sind. Westeuropa hat also gegenüber Japan Vorteile ebenso wie gegenüber den USA, die innerhalb der NAFTA extrem unterschiedliche Wirtschaften (Kanada, Mexiko und die USA) za einem kohäsiven System vereinigen müssen. In den USA beträgt das Pro-KopfEinkommen 22.000 US\$, in Mexiko 2.680 US\$; der Stundenlohn in der gewerblichen Wirtschaft beträgt im US-Durchschnit 1.4,77 US\$, in Mexiko gerade 1,80 US\$. Bei diesen Unterschieden ist die Art der Arbeitsteilung entlang der Lohnkosten 
im nordamerikanischen Wirtschaftsraum vorgezeichnet. Für Japan ist der pazifische Raum im wesentichen eine Aneinanderreihung von »Syntropieinseln«, aus denen Rohstoffe, Energie und Arbeitskuäfte entnommen werden, um sie unter japanischer Disposition höchst effizzient in den modernsten Stoff- und Energietransformationssystemen zu Produkten zli verarbeiten, die den gesanten Weltmarkt iiberschwemmen.

Die regionalen Blöcke bilden nur eine Seite der Entwicklungstendenzen der modernen Weltwirtschaft ab. Denn sowohl die nationalen Räume als auch das globale System insgesant existieren weiter. Die Bildung der strategischen Allianzen zwischen Unternehmen aus den USA und Japan, aus der Bundestepublik und den USA oder Japan, die Bildung von europaweiten Konsortien mit der Beteiligung USamerikanischer Fimen etc. deuten darauf hin, daß für High-Tech-Produkte nationale und selbst integrierte Blockmärkte zu klein sind, als daß sie rentabel beliefert werden könnten. Die Expansionskraft des ökonomischen Prinzips im Kapitalismus hat die nationalen Grenzen gesprengt und auf diese Weise ihren Beitrag zur Blockbildung geleistet. Sie weist aber auch über die Blöcke hinaus und unterstreicht die Relevanz und Virulenz des Kapitalismus als eines globalen Systems.

Das Ausspielen der globalen Karte ist jedoch am Ende des 20. Jahxhun- derts keineswegs gleichbedeutend mit der verstaubten Option des Freihandels gemäß dem Theorem der komparativen Kostenvorteile des David Ricardo. Denn wenn der Wein und das Tuch, also die beiden Produkte, an denen Ricardo sein Theorem exemplifizierte, innerhalb des gleichen transnational operierenden Konzerns produziert werden sollten, macht der Ansatz keinen Sinn. Da heute immerhin 50 bis 60 Prozent des Welthandels Intrakonzernhandel sind, da »administered prices « und nicht frei gebildete Marktpreise fakturiert werden, kann durch den Marktmechanismus das (unterstellte) Wohlfahrtsoptimum des freien Handels nicht mehr garantiert werden. Also haben die okonomischen Tendenzen von Akkumulation und Expansion über die Grenzen hinaus, haben die Konzentration und Zentralisation selbst ihren Beitrag dazu geleistet, daß die Wirkung der Preismechanismen auf den Weltmärkten nur unvollkommen ist. Politische Interventionen erlangen größere Bedeutung. Wenn schon die ökonomischen Einheiten, die transnationalen oder multinationalen Konzerne, über die Grenzen hinaus operieren, die der Nationalstaat setzt, muß auch der Stat seine Funktionen trans- und internationalisieren (vgl. dazu Rolf Knieper, John Holloway und Sol Picciotto in diesem Heft). Wenn wir die imperialistische Expansion des späten 19. Jahrhunderts am Ende des 20. Jahrhunderts ausschließen, dann 
müssen Formen der Kooperation mit anderen Nationalstaaten gesucht werden. Dies ist immer gleichbedeutend mit der Übertragung von Souveränitätsrechten des Nationalstaats an supranationale Einrichtungen - ein Erfordernis, das in diesem Heft Uli Jahner unterstreicht.

»Staatlichkeit « fallt also nicht mehr mit »Nationalstaatlichkeit« zusammen.

Daß den Wirtschaftsbö̈cken informelle und - zumindest in Falle der $\mathrm{EG}$ - formelle Souveränitütsrechte zufallen, ist offensichtlich. Doch auch »unterhalb « der Ebene des traditionellen Nationalstaats ergeben sich tiefgreifende Veränderungen. Mit dem Bedeutungszuwachs der supranationalen Institutionen und dem Bedeutungsverlust nationalstaatliclıer Souveränität steigt natürlich auf der anderen Seite auch das Gewicht der Regionen innerhalb von traditionellen Nationalstaaten. Dies kann so weit gehen, daß neue regionale Gruppierungen über nationalstaatliche Grenzen himweg cntstehen, die in der Tendenz zur Auflösung der tradierten nationalstaatlichen Gebilde führen können. Der Regionalismus in der EG von Norditalien bis Wallonien, von Katalanien bis Schottland ist bislang nur die Schwalbe, die aber einen (unfreundlichen) Sonmer machen kamn. Auch anderswo gibt es Tendenzen dieser Art: In Kanada die »cleavages« zwischen dem pazifischen Westen und dem atlantischen Osten, zwischen dem francophonen und dem anglophonen Teil des Landes. In Kalifornien gibt es regionalistische Tendenzen, und selbst in Brasilien gedeiht der Separatismus, weil die ökonomischen Verbindungen des Südens mit den Stataten des »Merco Sur dichter sind als die Beziehungen innerhalb der Nation mit dem armen Norden oder Nordosten. Ökonomische Tendenzen transformieren sich in politische Interessen und Konflikte. Mit anderen Worten: Die Blockbildung in der Weltwirtschaft restrukturiert nicht nur das kapitalistische Weltsystem sondern tendenziell auch dessen $»$ Regionen

Wenn von der Triade Westeuropas, Nordamerikas und des pazifischen Raums die Rede ist, wird die ehemals so genannte »Dritte Welt" ebenso vergessen wie Zentraleuropa, Osteuropa und die Nachfolgestataten der Sowjetunion. Die Dritte Welt ist marginalisiert, ihre ökonomische Bedeutung auf fast Null reduziert, es sei denn, daß einzelne Staaten oder Regionen über wichtige energetische und materiale Rohstoffe verfügen, so wie die Länder in Nahen Osten. Die große Mehrheit der Weltbevölkerung ist an den Rand gedrängt und aus dem Weltgeschehen ausgeblendet. $\mathrm{Ob}$ die $\gg$ Exklusion« von nahezu $80 \%$ der Weltbevölkerung aus dem Weltmarkt der Industrieländer und blöcke auf die Dauer durchgehalten werden kann, darf bezweifelt werden. Der Verabschiedung ganzer Kontinente aus der Welpolitik folgt 
mit Sicherheit die Rückmeldung und sei es nur mit Katastrophen, deren Vorboten derzeit in Somalia, Zaire, Angola oder im Sudan eine neue Kategorie von Hilfe za provozieren scheinen: »humanitär-militärisches Engagement«. Ganze Völker werden zum Spielmaterial der vorherrschenden ökonomischen und politischen Mächte degradiert. Den sich stabilisicrenden Blöcken korrespondiert eine gefährliche Destabilisierung an den »Rändern« der Triade.

Und der Osten? Zunächst scheint es, als ob sich ein neuer Markt von 290 Millionen ehemaligen Sowjetbürgern plus 125 Millionen Osteuropäern auftun könnte. »Perhaps we could consider the commonwealth as a kind of new frontier Jude Wamninski in Foreign Affains (Spring 1992, 25) über 'Die Zukunft Rußlands'. Über 400 Millionen potentielle Konsumenten sind kein Pappenstiel. Sie könnten der wellwirtschaftlichen Entwicklung einen kräftigen Impuls geben, vorausgesetzt den osteuropäischen Transformationen (dazu vgl. Heft 89 der PROKLA) gelänge in überschaubaren Fristen ein Anschluß an die westliche Modemisierung. Wenn jedoch Osteuropa und die GUS in absehbarer Zeil keine Chance haben, sich als ein selbständiges Zentrum, als ein regionaler Block in der einen oder anderen Weise innerhalb der Weltwirtschaft zu formieren, dann bleibt noch der Rohstoffreichtum des asiatischen
Teils der ehemaligen Sowjetunion. Er wird zu einem neuen Objekt der Begierde, das die industriellen Stoff- und Energiewandlungssysteme in den Zentren der Triade mit Nachschub versorgen könnte. Wenn dem so ist, stellt sich die entscheidende Frage, ob Osteuropa und die GUS za einer Art »Almende« der drei Blöcke der Triade werden, oder ob zwischen ihnen ein Konkurrenzkampf um EinfluB in Osteuropa und der GUS ausbrechen wird. - Lenin hat zwar mit seiner Revolutionstheorie in exorbitanter Weise historisch Unrecht bekommen; aber mit seiner Analyse der imperialistischen Konkurrenz könnte er ebenso grandios und noch dazu auf dem Territorium der ehemaligen Sowjetunion Recht behalten...

PROKLA-Redaktion 